\title{
A Damping Ring Design for the SLAC Next Linear Collider*
}

T. O. Raubenheimer, J. Byrd, J. Corlett, R. Early, M. Furman, A. Jackson, P. Krejcik, K. Kubo, T. Mattison, M. Minty, W. Moshammer, D. Robin, B. Scott, J. Spencer, K. Thompson, P. Wilson Stanford Linear Accelerator Center, Stanford University, Stanford, CA, 94309

Lawrence Berkeley Laboratory, Berkeley, CA, 94720

\section{Abstract}

In this paper, we describe the design of the main damping rings and the positron pre-damping ring for the SLAC Next Linear Collider, a future linear collider with a centerof-mass energy of 0.5 to $1.5 \mathrm{TeV}$. The rings will operate at an energy of $2 \mathrm{GeV}$ with a maximum repetition rate of $180 \mathrm{~Hz}$. The normalized extracted beam emittances are $\gamma \epsilon_{x}=3 \mathrm{~mm}$-mrad and $\gamma \epsilon_{y}=0.03 \mathrm{~mm}$-mrad. To provide the necessary damping, the rings must damp multiple trains of bunches. Thus, the beam current is large, roughly $1 \mathrm{~A}$. We will present the optical layout, magnet designs, and RF systems, along with the dynamic aperture and required alignment tolerances; collective effects will be discussed in another paper.

\section{INTRODUCTION}

The primary requirements of the NLC damping rings are summarized in Table 1 for two stages of the NLC design [1]. The rings must produce electron and positron beams with emittances of $\gamma \epsilon_{x}=3 \mathrm{~mm}$-mrad and $\gamma \epsilon_{y}=$ $0.03 \mathrm{~mm}$-mrad at a repetition rate as high as $180 \mathrm{~Hz}$. The beams in the rings consist of trains of as many as 90 bunches, spaced by $1.4 \mathrm{~ns}$, with a maximum single bunch population of $1.5 \times 10^{10}$.

Table 1. Damping ring requirements for NLC designs.

\begin{tabular}{|c|c|c|}
\hline & NLC-I $(500 \mathrm{GeV})$ & NLC-III $(1.5 \mathrm{TeV})$ \\
\hline$\gamma \epsilon_{x}$ & $3 \times 10^{-6} \mathrm{~m}$-rad & $3 \times 10^{-6} \mathrm{~m}$-rad \\
\hline$\gamma \epsilon_{y}$ & $3 \times 10^{-8} \mathrm{~m}$-rad & $3 \times 10^{-8} \mathrm{~m}$-rad \\
\hline Rep. Rate & $180 \mathrm{~Hz}$ & $120 \mathrm{~Hz}$ \\
\hline Bunch Charge & $0.7 \times 10^{10}$ & $1.5 \times 10^{10}$ \\
\hline Bunch Sep. & $1.4 \mathrm{~ns}$ & $1.4 \mathrm{~ns}$ \\
\hline Bunches/Train & 90 & 75 \\
\hline
\end{tabular}

The NLC electron injector is based on a polarized photocathode in a DC gun with a subharmonic buncher system. It is designed to produce bunch trains with a normalized emittance of $\gamma \epsilon_{x, y}=100 \mathrm{~mm}$-mrad and a momentum spread less than $\pm 1 \%$. Thus, the electron vertical emittance must be damped by roughly four orders of magnitude. Since the damping ring must operate uncoupled to produce the flat beams, this specifies the required number of vertical damping times; assuming a vertical equilibrium emittance of $2 \times 10^{-8} \mathrm{~m}$-rad, the beams must be stored for 4.6 vertical damping times.

*Work supported by the Department of Energy, contracts DEAC03-76SF00515 (SLAC) and DE-AC03-76SF00098 (LBL).
Thus, the primary design problems in the rings involve attaining both very fast damping and very small equilibrium emittances with a large dynamic aperture since the beam emittances are large at injection. Simple scaling shows that these competing requirements would force one to a large circumference ring which can store and damp many trains of bunches at the same time [2].

The situation is even worse in the case of the positron injector, where to produce the required number of positrons, the captured positron beam emittance is large; in the NLC design [3], the incoming positrons have an emittance that is roughly 600 times the incoming electron emittance and an energy spread of $\pm 2 \%$. In this case, it would be extremely difficult to attain the required damping and the necessary dynamic aperture in a single ring. Therefore, we will use a pre-damping ring to damp the incoming positron emittance to the level of the incoming electron emittance; the positrons can then be transfered to the main damping ring which would be identical to the electron damping ring. Assuming a normalized equilibrium emittance of $30 \mathrm{~mm}$-mrad in the pre-damping ring, the positron beams must be damped for 3.2 damping times to attain extracted rms emittances equal to that of the incoming electron bearns.

Table 2. Pre-damping ring parameters.

\begin{tabular}{|c|c|}
\hline Energy & $1.8 \sim 2.2 \mathrm{GeV}$ \\
\hline Circumference & $112 \mathrm{~m}$ \\
\hline Current & $1 \mathrm{Amp}$ \\
\hline$\nu_{x}, \nu_{y}, \nu_{s}$ & $10.18,4.18,0.015$ \\
\hline$\gamma \epsilon_{x, y}$ (fully coupled) & $2.5 \times 10^{-5} \mathrm{~m}-\mathrm{rad}$ \\
\hline$\sigma_{\epsilon}, \sigma_{z}$ & $0.1 \%, 7.0 \mathrm{~mm}$ \\
\hline$\tau_{x}, \tau_{y}, \tau_{z}$ & $3.0 \mathrm{~ms}, 4.0 \mathrm{~ms}, 2.4 \mathrm{~ms}$ \\
\hline$V_{R F}, f_{R F}$ & $1.5 \mathrm{MV}, 714 \mathrm{MHz}$ \\
\hline
\end{tabular}

Table 3. Main damping ring parameters.

\begin{tabular}{|c|c|}
\hline Energy & $1.8 \sim 2.2 \mathrm{GeV}$ \\
\hline Circumference & $223 \mathrm{~m}$ \\
\hline Current & $1 \mathrm{Amp}$ \\
\hline$\nu_{x}, \nu_{y}, \nu_{s}$ & $23.81,8.62,0.004$ \\
\hline$\gamma \epsilon_{x}, \gamma \epsilon_{y}$ & $3 \times 10^{-6} \mathrm{~m}-\mathrm{rad}, 2 \times 10^{-8} \mathrm{~m}-\mathrm{rad}$ \\
\hline$\sigma_{\epsilon}, \sigma_{z}$ & $0.09 \%, 4.1 \mathrm{~mm}$ \\
\hline$\tau_{x}, \tau_{y}, \tau_{z}$ & $4.0 \mathrm{~ms}, 4.6 \mathrm{~ms}, 2.5 \mathrm{~ms}$ \\
\hline$V_{R F}, f_{R F}$ & $1.0 \mathrm{MV}, 714 \mathrm{MHz}$ \\
\hline
\end{tabular}

The principal parameters of the pre- and main damping 
rings are listed in Tables 2 and 3 . The nominal operating energy is $2.0 \mathrm{GeV}$, although the rings are being designed to operate between $1.8 \mathrm{GeV}$ and $2.2 \mathrm{GeV}$. This will provide the operational flexibility to balance faster damping rates against smaller equilibrium emittances. Both rings will store multiple trains of bunches. The pre-damping ring will store two 90 bunch trains at once while the main damping ring will store four 90 bunch trains. The bunches in a train are separated by 1.4 ns while the trains themselves are separated by 60 ns so that fast kickers can inject and extract individual trains. In this manner, each bunch train is stored for two machine cycles in the pre-damping ring and four machine cycles in the main damping ring. Finally, the maximum average current is roughly $1 \mathrm{~A}$ for the NLC-III parameters; note that for NLC-III the bunch trains are only 75 bunches long and the train separation is roughly $80 \mathrm{~ns}$.

The pre-damping ring will operate on the difference coupling resonance. In this case, the equilibrium emittances are $\gamma \epsilon_{x, y}=30 \mathrm{~mm}$-mrad and the transverse damping time is $\tau_{x, y}=3.45 \mathrm{~ms}$; thus, after two $180 \mathrm{~Hz}$ machine cycles, the beams are stored for 3.2 damping times. In the main damping ring, the horizontal equilibrium emittance, including the intrabeam scattering for $1.5 \times 10^{10}$, is $\gamma \epsilon_{x}=3.1 \mathrm{~mm}-\mathrm{mrad}$ while the vertical equilibrium emittance of $\gamma \epsilon_{y}=0.02 \mathrm{~mm}$-mrad is determined by the alignment tolerances. Finally, the vertical damping time is 4.64 $\mathrm{ms}$; this corresponds to roughly 4.8 vertical damping times. In both rings, the damping is slightly greater than that required; this provides a margin for injection transients and mismatches.

In the next sections, we will briefly describe the optical design of the positron pre-damping ring and the main damping rings. Then, we will discuss the injection/extraction and the RF systems. Finally, we will describe the tolerances and dynamic aperture of the rings.

\section{OPTICAL DESIGNS}

Both rings are designed in a race-track form with two arcs separated by straight sections. The pre-damping ring is roughly 110 meters in circumference while the main damping ring is twice as large.

In the pre-damping ring, each arc consists of 14 FOOF cells plus dispersion matching sections; the FOOF cell is a modified FODO lattice where the defocusing quadrupole is replaced by a combined function bending magnet. Because the ring needs a large dynamic aperture and does not require a small equilibrium emittance, we use a weak focusing lattice. We chose to use the FOOF structure since it constrains the beta functions, allowing us to design a smaller vacuum chamber aperture and thereby less expensive magnets. In the arcs, the vacuum chamber measures $3 \mathrm{~cm}$ by $3.2 \mathrm{~cm}$. This provides physical aperture for an injected normalized edge emittance of $0.09 \mathrm{~m}$-rad $(50 \%$ larger than nominal) plus $2 \mathrm{~mm}$ clearance for alignment and steering.

In the main damping ring, each arc consists of 19 TME cells [4] plus dispersion matching sections. The straight sections are roughly 30 meters in length. One side of the ring is devoted to the damping wigglers while the other side contains the injection and extraction components and the RF cavities.

The TME cells consist of a single combined function bending magnet, two focusing quadrupoles and a single defocusing quadrupole. Each cell contains six sextupoles, in three families, to correct the chromaticity. The vacuum chamber is circular with a $25 \mathrm{~mm}$ diameter and an antechamber to handle the intense synchrotron radiation; the chamber is described further in Ref. [5]. Preliminary designs have been made of the bending magnets, quadrupoles and sextupoles. The bending magnet has a central field of $15.3 \mathrm{kG}$ with a gradient of $125 \mathrm{kG} / \mathrm{m}$ and a half gap of $1.6 \mathrm{~cm}$. The quadrupoles have a maximum gradient of 600 $\mathrm{kG} / \mathrm{m}$ with an aperture of $1.6 \mathrm{~cm}$ while the sextupoles have a maximum gradient $\partial^{2} B_{y} / \partial x^{2}$ of $30,000 \mathrm{kG} / \mathrm{m}^{2}$ with an aperture of $1.7 \mathrm{~cm}$. In all cases, the poles are designed to fit around the ante-chamber.

In addition, the main damping ring requires roughly 25 meters of high field wiggler to attain the desire damping times. The two parameters that are relevant for a damping wiggler are the integral of $B_{y}^{2}$, which determines the damping, and the quantum excitation, which is set by the field and the period. In the NLC design, we have chosen to consider a relatively short, high field device. If we assume a sinusoidal $B_{y}$ with a peak of $22 \mathrm{kG}$ (close to the saturation of Vanadium Permandur), then we need a length of 25.6 meters and a period of $25 \mathrm{~cm}$. Simple scaling laws suggest that such a wiggler could be build as either a permanent magnet hybrid wiggler or an electromagnetic wiggler.

The SSRL Beam-Line 9 wiggler [6], which was recently constructed, nearly meets our requirements. It is a hybrid wiggler with a peak field of $20.5 \mathrm{kG}$ and a $26 \mathrm{~cm}$ period. Because the wiggler poles were designed to optimize the total flux, the field is not sinusoidal and the integral of $B_{y}^{2}$ is within $1 \%$ of our requirements.

\section{INJECTION/EXTRACTION}

In both damping rings, injection and extraction are performed using DC septum magnets and pulsed kicker magnets. The design concept is similar to the system developed for the SLC [7]. The kickers are required to inject or extract a single $126 \mathrm{~ns}$ bunch train onto or from the closed orbit without disturbing the other stored bunches. Thus the rise and the fall times must be less than the train separation $60 \mathrm{~ns}$. Finally, to reduce RF transients, a new bunch train is injected on the same revolution that a damped bunch train is extracted.

The main damping ring kickers must provide a deflection of 2.5 mrad with a stability of $\Delta \theta / \theta \leq 0.5 \times 10^{-3}$ for the extraction kickers and $\Delta \theta / \theta \leq 3.5 \times 10^{-3}$ for the injection kickers; these tolerances limit the beam jitter due to the kickers to $10 \%$ of the beam size. The deflection can be provided using a 1.2 meter kicker with an impedance of $50 \Omega$ and a voltage of $17 \mathrm{kV}$. Achromatic (double) kicker systems will be used to ease the stability requirements. Here, an identical kicker, powered by the same pulser, is placed in the injection/extraction line and separated from 


\section{DISCLAIMER}

This report was prepared as an account of work sponsored by an agency of the United States Government. Neither the United States Government nor any agency thereof, nor any of their employees, make any warranty, express or implied, or assumes any legal liability or responsibility for the accuracy, completeness, or usefulness of any information, apparatus, product, or process disclosed, or represents that its use would not infringe privately owned rights. Reference herein to any specific commercial product, process, or service by trade name, trademark, manufacturer, or otherwise does not necessarily constitute or imply its endorsement, recommendation, or favoring by the United States Government or any agency thereof. The views and opinions of authors expressed herein do not necessarily state or reflect those of the United States Government or any agency thereof. 


\section{DISCLAIMER}

Portions of this document may be illegible in electronic image products. Images are produced from the best available original document. 
the kicker in the ring by a horizontal $\pm I$ transform. Finally, to reduce the rise and fall times requirements, the injection and extraction kickers in the ring are also separated by $a \pm I$ transform in the horizontal plane; the rising and falling kicker pulses can then be tuned to compensate the deflections to the stored beams.

In the pre-damping ring, because of the large beam emittances, the required deflection is roughly three times larger and the required kicker aperture is roughly $50 \%$ larger than in the main damping ring. Fortunately, the stability requirements are relaxed by a factor of two. To maintain conservative magnet and pulser designs, both the injection and the extraction kickers would consist of four $50 \mathrm{~cm}$ magnets powered in parallel.

\section{RF SYSTEMS}

The RF parameters are summarized in Tables 2 and 3. Both rings will use $714 \mathrm{MHz}$ systems; this is the lowest frequency that is consistent with the $1.4 \mathrm{~ns}$ bunch spacing. Because of the long bunch trains, the RF cavities must have HOM damping. At this stage of the design, we have assumed scaled versions of the PEP-II single-mode RF cavities [8]; further discussion can be found in Ref. [5].

In the pre-damping ring, the required RF voltage is determined by the size of the RF bucket. The incoming beam is expected to have a momentum deviation of $\pm 2 \%$. This would require an RF voltage of $2.5 \mathrm{MV}$. Instead, an energy compressor, located at the entrance of the ring, will reduce the incoming energy deviations by a factor of two. Thus, we plan to use an RF voltage of $1.5 \mathrm{MV}$; this provides an energy bucket of $1.5 \%$.

In the main damping ring, the RF voltage is determined by the energy loss per turn. The synchrotron radiation loss is $635 \mathrm{keV} /$ turn and we have estimated the parasitic losses at $120 \mathrm{keV} /$ turn by scaling from the old PEP ring. This is felt to be a conservative estimate; scaling from the ALS or the PEP-II rings suggest parasitic losses an order of magnitude smaller. In this case, a $1 \mathrm{MV}$ RF voltage would be sufficient. It provides sufficient voltage to prevent the beam loading instability while allowing for a longer bunch length which is desirable.

\section{TOLERANCES AND DYNAMIC APERTURE}

The tolerances in the pre-damping ring are determined by the requirements on the dynamic aperture while the tolerances in the main. damping rings are dominated by the requirements on the vertical equilibrium emittance. In the pre-damping ring, the bare ring (without errors) has a normalized acceptance of $\gamma A \leq 0.45 \mathrm{~m}$-rad over $\delta p / p= \pm 2 \%$; the acceptance is $A_{x} \equiv \frac{1}{2}\left(\gamma x^{2}+2 \alpha x x^{\prime}+\beta{x^{\prime}}^{2}\right)$. With random transverse alignment, rotation, and strength errors of $200 \mu \mathrm{m}, 1 \mathrm{mrad}$, and $0.1 \% \mathrm{rms}$ on all elements, the acceptance is reduced to $0.2 \mathrm{~m}$-rad. This is still a factor of two larger than the physical aperture.

In the main damping ring, the primary limitation arises from the vertical dispersion generated by misalignments of the sextupoles and rotation errors of the strong focusing quadrupoles. The alignment tolerances required to attain an equilibrium emittance of $2 \times 10^{-8} \mathrm{~m}$-rad are listed in Table 4. These tolerances assume the use of four skew quadrupoles to corrected the vertical dispersion errors.

Table 4. Alignment tolerances in the main damping ring.

\begin{tabular}{|c|c|}
\hline Sextupole $(X / Y / \Theta)[\mu \mathrm{m} / \mathrm{mrad}]$ & $100 / 50 / 1$ \\
\hline Arc QFs $(X / Y / \Theta)[\mu \mathrm{m} / \mathrm{mrad}]$ & $100 / 100 / 0.6$ \\
\hline Other Quads. $(X / Y / \Theta)[\mu \mathrm{m} / \mathrm{mrad}]$ & $100 / 100 / 1$ \\
\hline Bends $(X / Y / \Theta)[\mu \mathrm{m} / \mathrm{mrad}]$ & $100 / 100 / 0.6$ \\
\hline BPMs $(X / Y / \Theta)[\mu \mathrm{m} / \mathrm{mrad}]$ & $100 / 100 / 1$ \\
\hline
\end{tabular}

The tolerances for the dynamic aperture are looser. Doubling the nominal alignment tolerances listed in Table 4 still yields an aperture in excess of $3 \sigma$ for an injected beam with ten times the nominal injected emittance and a relative momentum deviation between $\pm 1 \%$.

Obviously, both rings have severe alignment tolerances. To attain the required values, we plan to use a beam-based alignment technique where trims on the individual magnets are varied and the resulting orbit deflections are used to infer the magnet centers. Similar techniques are being used successfully at the ALS and LEP storage rings.

\section{DISCUSSION}

In this paper, we have presented the design of the SLAC NLC damping rings. Both a large aperture predamping ring and a main damping ring are needed to damp the positron beam while a single ring is used for the electron beam. The extracted emittances of the rings are $\gamma \epsilon_{x}=3 \mathrm{~mm}$-mrad and $\gamma \epsilon_{y}=0.03 \mathrm{~mm}$-mrad. We have discussed the optical designs, the RF systems, the injection/extraction kickers, and the alignment tolerances and dynamic aperture. Further discussion of collective effects can be found in Ref. [5].

\section{References}

[1] T. O. Raubenheimer, et. al., "Parameters of the SLAC Next Linear Collider," these proceedings.

[2] T. O. Raubenheimer, R. D. Ruth, and L. Z. Rivkin, "Damping Ring Designs for TeV Linear Colliders," Proc. of the DPF Summer Study, Snowmass '88, Snowmass, CO (1989).

[3] H. Tang, et. al., "The NLC Positron Source," these proceedings.

[4] L. C. Teng, "Minimum Emittance Lattice for Synchrotron Radiation Storage Rings," ANL-LS-17 and ANL-LS-17A (1985).

[5] T. O. Raubenheimer, et. al., "Collective Effects in the SLAC NLC Damping Ring Designs," this conference.

[6] M. Baltay, private communication (1995).

[7] T. Mattison, et. al., "Status of the SLC Damping Ring Kicker Systems," Proc. 1991 Part. Acc. Conf., San Francisco, CA (1991).

[8] Rimmer, R. A., "RF Cavity Development for the PEPII B-Factory," Proc. Int. Workshop on B-Factories, KEK, Tsukuba, Japan (1992). 